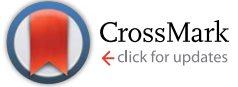

Cite this: J. Mater. Chem. A, 2017, 5 , 1567

Received 26th October 2016 Accepted 4th December 2016

DOI: $10.1039 / c 6 t a 09278 \mathrm{k}$

www.rsc.org/MaterialsA

\section{An interfacial engineering approach towards two- dimensional porous carbon hybrids for high performance energy storage and conversion $\uparrow$}

\author{
Chenbao Lu, ${ }^{\text {ab }}$ Shaohua Liu, ${ }^{\mathrm{b}}$ Fan Zhang, ${ }^{\text {b }}$ Yuezeng Su, ${ }^{\star a}$ Xiaoxin Zou, ${ }^{\mathrm{c}}$ Zhan Shi, ${ }^{\mathrm{c}}$ \\ Guodong $\mathrm{Li}^{\mathrm{C}}$ and Xiaodong Zhuang*bd
}

In order to improve the performance and fundamental understanding of conducting polymers, development of new nanotechnologies for engineering aggregated states and morphologies is one of the central focuses for conducting polymers. In this work, we demonstrated an interfacial engineering method for the rational synthesis of a two-dimensional (2D) polyaniline (PANI) nano-array and its corresponding nitrogen-doped porous carbon nanosheets. Not only was it easy to produce a sandwichlike 2D morphology, but also the thickness, anchored ions and produced various metal phosphides were easily and rationally engineered by controlling the composition of the aqueous layer. The novel structural features of these hybrids enabled outstanding electrochemical capacitor performance. The specific capacitance of the as-produced diiron phosphide embedded nitrogen-doped porous carbon nanosheets was calculated to be as high as $1098 \mathrm{~F} \mathrm{~g}^{-1}$ at $1 \mathrm{~A} \mathrm{~g}^{-1}$ and an extremely high specific capacitance of $611 \mathrm{~F} \mathrm{~g}^{-1}$ at $10 \mathrm{~A} \mathrm{~g}^{-1}$, outperforming state-of-the-art performance among porous carbon and metal-phosphide-based supercapacitors. We believe that this interfacial approach can be extended to the controllable synthesis of various 2D material coupled sandwich-like hybrid materials with potential applications in a wide range of areas.

\section{Introduction}

Since the discovery of conducting polymers, polyaniline (PANI) has captured the attention of the scientific community because of its unique chemical structure, optoelectronic properties and wide applications. ${ }^{1}$ Given that the nano-architecture of materials plays a crucial role in achieving the promising fundamental properties. Over the past decade, a lot of synthetic strategies toward PANIs with different doping states ${ }^{2}$ and various nanostructures, such as nanospheres, ${ }^{3}$ nanofibers, ${ }^{4}$ nanotubes, ${ }^{5}$ and nanosheets, ${ }^{6}$ have been extensively developed due to the urgent requirements in optoelectronic and energy

${ }^{a}$ School of Chemistry and Chemical Engineering, State Key Laboratory of Metal Matrix
Composites, 200240 Shanghai, P. R. China. E-mail: yzsu@sjtu.edu.cn
${ }^{b}$ Shanghai Key Lab of Electrical Insulation and Thermal Ageing, Shanghai
Electrochemical Energy Devices Research Center, School of Chemistry and Chemical
Engineering, Shanghai Jiao Tong University, 200240 Shanghai, P. R. China. E-mail:
fan-zhang@sjtu.edu.cn; zhuang@sjtu.edu.cn
${ }^{c}$ State Key Laboratory of Inorganic Synthesis and Preparative Chemistry, International
Joint Research Laboratory of Nano-Micro Architecture Chemistry, College of
Chemistry, Jilin University, Changchun 130012, P. R. China
${ }^{d}$ Center for Advancing Electronics Dresden (cfaed), Department of Chemistry and Food
Chemistry, Technische Universität Dresden, Mommsenstrasse 4, Dresden 01062,
Germany $\dagger$ Electronic supplementary information (ESI) available. See DOI: $10.1039 / \mathrm{c} 6 \mathrm{ta} 09278 \mathrm{k}$ related fields. ${ }^{2,7}$ However, most of the methods for nanostructured PANI production can only be carried out in homogeneous solution with the aid of vigorous stirring, heating or high pressure, suffering from complicated procedures and a difficult-to-control morphology. ${ }^{7}$ It has long been noticed that the interfacial method can be used to prepare PANI and its composites. $^{2,8}$ However, synthesis of conducting polymers with a controllable aggregation state and morphology through an interfacial approach still remains great challenge.

Two-dimensional (2D) nanomaterials have attracted tremendous attention since the discovery of graphene. Of these, 2D soft nanomaterials, ${ }^{9}$ such as graphene-coupled conducting polymer nanosheets, 2D sandwich-like conjugated microporous polymers and other types of 2D materials, have been studied and used as crucial materials for many optoelectronic and energy applications in the past few years, ${ }^{\mathbf{1 0}}$ for example, as porous materials for sensing, gas storage and separation, and as precursors for the preparation of 2D hybrid materials for energy storage and conversion. Considerable efforts have been made to prepare graphene-based 2D hybrid materials such as composites of graphene-based transition metals, metal oxides, metal chalcogenides, and metal phosphides (MPs). ${ }^{\mathbf{1 1}}$ Besides, both metal supported and free-standing chemical vapor deposition (CVD) graphene have been used as templates to fish nanoparticles from organic/aqueous interfaces to prepare asymmetric $\mathrm{MoS}_{2}$ /graphene/metal sandwiches and noble metal 
particles loaded graphene, respectively. ${ }^{12}$ However, due to the general poor compatibility between graphene and substantially dissimilar precursors, the controlled growth of small nanoparticles on graphene surfaces still relies on varied and complicated synthetic procedures. ${ }^{13}$

Herein, an interfacial approach was successfully developed for the fast preparation of a graphene-coupled polyaniline nanoarray with a uniform 2D morphology and in large quantity. Not only can a sandwich-like 2D morphology be easily produced, but the thickness and shape of PANI nano-array, as well as the anchored transition metal cations and acid anions of the asprepared nanosheets can also be engineered rationally. Consequently, metal phosphide-embedded nitrogen-doped porous carbon nanosheets (denoted as RG@(MP $\subset$ NPC)) can be easily prepared by direct pyrolysis of the as-prepared ionanchored 2D PANI nano-array. Notably, the as-prepared diiron phosphide-embedded NPC (RG@( $\left.\mathrm{Fe}_{2} \mathrm{P} \subset \mathrm{NPC}\right)$ ), which has an Fe content of only $3.71 \mathrm{wt} \%$ and a specific surface area of $240 \mathrm{~m}^{2}$ $\mathrm{g}^{-1}$, exhibits ultrahigh specific capacitances of up to $1098 \mathrm{~F} \mathrm{~g}^{-1}$ at $1 \mathrm{~A} \mathrm{~g}^{-1}$ and $611 \mathrm{~F} \mathrm{~g}^{-1}$ at $10 \mathrm{~A} \mathrm{~g}^{-1}$, which are more than ten times the values for NPC, $\mathrm{Fe}_{2} \mathrm{P}$, and RG@NPC. Thus, it outperforms state-of-the-art supercapacitors based on porous carbons and metal phosphide-based materials. By replacing the metal ions in the aqueous layer, other kinds of MP and dual MP embedded carbon nanosheets can be easily prepared. Such interfacial engineering approach offers a new way for the design and preparation of novel 2D hybrid materials for high performance energy storage and conversion.

\section{Experimental}

\section{Chemicals}

$\mathrm{FeCl}_{3} \cdot 6 \mathrm{H}_{2} \mathrm{O}, \mathrm{CoCl}_{2} \cdot 6 \mathrm{H}_{2} \mathrm{O}, \mathrm{Ni}(\mathrm{OAc})_{2} \cdot 4 \mathrm{H}_{2} \mathrm{O},\left(\mathrm{NH}_{4}\right)_{2} \mathrm{Mo}_{4} \mathrm{O}_{13} \cdot 2 \mathrm{H}_{2} \mathrm{O}$ and aniline were purchased from Aladdin Reagent Co. Ammonium peroxydisulfate (APS), $\mathrm{HCl}(36 \mathrm{wt} \%), \mathrm{H}_{3} \mathrm{PO}_{4}(80 \%), \mathrm{H}_{2} \mathrm{SO}_{4}$ (98\%), $\mathrm{KMnO}_{4}, \mathrm{NaNO}_{3}$, and $\mathrm{H}_{2} \mathrm{O}_{2}$ were purchased from Sinopharm Chemical Reagent Co. Pt/C (20 wt\%) was purchased from Sigma-Aldrich. Nafion solution $(0.5 \mathrm{wt} \%)$ was purchased from DuPont, Ltd. All chemicals were used without further purification.

\section{Interfacial preparation of GO@PANI}

Typically, the interfacial reaction was performed in a $20 \mathrm{~mL}$ glass vial. First, ammonium peroxydisulfate (APS, $245 \mathrm{mg}$, $1 \mathrm{mmol}$ ) was dissolved in $8 \mathrm{~mL}$ of $1 \mathrm{M}$ dopant acid ( $\mathrm{HCl})$ solution. Then, $2 \mathrm{~mL}$ of aqueous GO dispersion $\left(2.5 \mathrm{mg} \mathrm{mL} \mathrm{mL}^{-1}\right)$ which was synthesized by a modified Hummers method was slowly added to the acid solution, followed by ultrasonication for $30 \mathrm{~min}$ to form a homogeneous suspension, as the aqueous layer. Subsequently, $100 \mu \mathrm{L}$ aniline was dissolved in the organic phase $(10 \mathrm{~mL})$ toluene, as the organic layer. Then, the organic phase was added carefully on top of the aqueous solution, forming an organic/aqueous interface. The steady interface was left to stand for 6 hours at room temperature. The graphene oxide-coupled polyaniline nanosheets (GO@PANI, $1: 20$ ) was obtained after being centrifuged and washed with DI water and ethanol. The GO@PANI $(1: 10)$ and GO@PANI $(1: 40)$ were synthesized by the same procedure with a different weight ratio of GO to aniline.

For the preparation of boric acid $\left(\mathrm{H}_{3} \mathrm{BO}_{3}\right)$ doped PANI nanosheets (GO@(B $\subset$ PANI)), phosphoric acid $\left(\mathrm{H}_{3} \mathrm{PO}_{4}\right)$ doped PANI nanosheets $\left(\mathrm{GO} @(\mathrm{P} \subset \mathrm{PANI})\right.$ ) and telluric acid $\left(\mathrm{H}_{6} \mathrm{TeO}_{6}\right)$ doped PANI nanosheets (GO@(Te $\subset$ PANI)), the same procedure was employed by just using $\mathrm{H}_{3} \mathrm{BO}_{3}, \mathrm{H}_{3} \mathrm{PO}_{4}$ and $\mathrm{H}_{6} \mathrm{TeO}_{6}$ as the doping acids, respectively.

\section{Preparation of metal phosphide anchored $\mathrm{N}$-doped porous carbon nanosheets}

Typically, $\mathrm{Fe}^{3+}$ and $\mathrm{PO}_{4}{ }^{3-}$ anchored PANI nanosheets (GO@(Fe) $\mathrm{P} \subset \mathrm{PANI})$ ) were first prepared by $\mathrm{FeCl}_{3}$ and $\mathrm{H}_{3} \mathrm{PO}_{4}$ involved interfacial polymerization. First, $245 \mathrm{mg}$ APS, $2 \mathrm{~mL}$ GO solution, and $1.5 \mathrm{~g} \mathrm{FeCl}_{3} \cdot 6 \mathrm{H}_{2} \mathrm{O}$ were added into $8 \mathrm{~mL}$ of $1 \mathrm{M}$ phosphoric acid aqueous solution. Subsequently, $100 \mu \mathrm{L}$ aniline was dissolved in $10 \mathrm{~mL}$ toluene as the organic layer. The organic phase was added carefully on top of the aqueous solution, forming an organic/aqueous interface. After reacting for $6 \mathrm{~h}$, GO@(Fe) $\mathrm{P} \subset \mathrm{PANI})$ can be obtained after drying. After thermal treatment of GO@(Fe $\mid \mathrm{P} \subset \mathrm{PANI})$ under a hydrogen/argon (5\%) atmosphere at $1000{ }^{\circ} \mathrm{C}$ for $2 \mathrm{~h}, \mathrm{RG} @\left(\mathrm{Fe}_{2} \mathrm{P} \subset \mathrm{NPC}\right)$ can be obtained.

The synthetic routes of $\mathrm{RG} @\left(\mathrm{Co}_{2} \mathrm{P} \subset \mathrm{NPC}\right), \mathrm{RG} @\left(\mathrm{Ni}_{12^{-}}\right.$ $\left.\mathrm{P}_{5} \subset \mathrm{NPC}\right)$, and $\mathrm{RG@(MoP \subset NPC)}$ are similar to that of RG@( $\left.\mathrm{Fe}_{2} \mathrm{P} \subset \mathrm{NPC}\right)$ by replacing $\mathrm{FeCl}_{3}$ to the corresponding transition metal salts $\left(\mathrm{CoCl}_{2}, \mathrm{Ni}(\mathrm{OAc})_{2}\right.$ and $\left.\left(\mathrm{NH}_{4}\right)_{2} \mathrm{Mo}_{4} \mathrm{O}_{13}\right)$. For the preparation of $\mathrm{Fe}_{2} \mathrm{P}$ and $\mathrm{Co}_{2} \mathrm{P}$ co-anchored NPC nanosheets (RG@( $\left.\left(\mathrm{Fe}_{2} \mathrm{P} \mid \mathrm{Co}_{2} \mathrm{P} \subset \mathrm{NPC}\right)\right)$, the same procedure was used by adding both $\mathrm{FeCl}_{3} \cdot 6 \mathrm{H}_{2} \mathrm{O}(800 \mathrm{mg})$ and $\mathrm{CoCl}_{2} \cdot 6 \mathrm{H}_{2} \mathrm{O}(800$ $\mathrm{mg}$ ) into the aqueous solution, as the dopants.

\section{Supercapacitor measurement}

The working electrodes were prepared by mixing the asprepared materials, carbon black (Super-P), and polytetrafluoroethylene (PTFE) at a weight ratio of $80: 10: 10$ and pressed on a platinum net. The area of the active material on each electrode was $\sim 1.0 \mathrm{~cm} \times 0.5 \mathrm{~cm}, \sim 0.5 \mathrm{mg}$ (RG@ $\left(\mathrm{Fe}_{2^{-}}\right.$ $\mathrm{P} \subset \mathrm{NPC}): 0.74 \mathrm{mg}, \mathrm{RG} @ \mathrm{NPC}: 0.45 \mathrm{mg}, \mathrm{NPC}: 0.60 \mathrm{mg}, \mathrm{Fe}_{2} \mathrm{P}$ nanoparticles: $2.46 \mathrm{mg}$ ). The electrochemical capacity of materials was evaluated in a three-electrode system, applying $6 \mathrm{M}$ $\mathrm{KOH}$ as the electrolyte and platinum wire and a $\mathrm{Ag} / \mathrm{AgCl}$ (saturated $\mathrm{KCl}$ ) electrode as the counter and reference electrodes, respectively. The electrochemical performance of samples was determined by cyclic voltammetry (CV) and galvanostatic charge-discharge. All electrochemical experiments were carried out at room temperature.

\section{Electrochemically catalysed ORR}

The electrodes were prepared as follows: $5 \mathrm{mg}$ catalyst was blended with $500 \mu \mathrm{l}$ Nafion solution (0.05 wt\%) and sonication for $2 \mathrm{~h}$, producing catalyst ink. Then $9 \mu \mathrm{l}$ catalyst ink was pipetted on the surface of a pre-polished glass carbon electrode $\left(0.2471 \mathrm{~cm}^{2}\right)$. The electrodes were dried at room temperature before measurement. The ORR experiments were carried out in a conventional three electrode cell using a Wave Driver 20 
bipotentiostatic (Pine Instrument Company, USA) at room temperature. $\mathrm{An} \mathrm{Ag} / \mathrm{AgCl}(\mathrm{KCl}, 3 \mathrm{M})$ reference electrode and a platinum wire counter electrode were used in the measurement. All tests were conducted in $0.1 \mathrm{M} \mathrm{KOH}$, and the potentials in this study refer to that of reversible hydrogen electrode (RHE). In $0.1 \mathrm{M} \mathrm{KOH}, E(\mathrm{RHE})=E(\mathrm{Ag} / \mathrm{AgCl})+0.944 \mathrm{~V}$. CV was performed in the potential range of $-0.95-0.05 \mathrm{~V} v s$. $\mathrm{Ag} / \mathrm{AgCl}$ reference electrode at a sweep rate of $100 \mathrm{mV} \mathrm{s}^{-1}$. RDE and RRDE were measured in $\mathrm{O}_{2}$-saturated $0.1 \mathrm{M} \mathrm{KOH}$ at $1600 \mathrm{rpm}$ at a sweep rate of $10 \mathrm{mV} \mathrm{s}^{-1}$.

The electron transfer number was determined by the following equation:

$$
n=4 I_{\mathrm{d}} /\left(I_{\mathrm{d}}+I_{\mathrm{r}} / N\right)
$$

The four electron selectivity of the catalysts was evaluated based on the $\mathrm{H}_{2} \mathrm{O}_{2}$ yield, calculated from the following equation:

$$
\mathrm{H}_{2} \mathrm{O}_{2}(\%)=200 I_{\mathrm{r}} / N\left(I_{\mathrm{d}}+I_{\mathrm{r}} / N\right)
$$

where $I_{\mathrm{d}}$ and $I_{\mathrm{r}}$ refer to disk current and ring current, respectively; and $N=0.37$ is the ring collection efficiency.

\section{Results and discussion}

The preparation of GO@PANI through in situ interfacial polymerization is illustrated in Fig. 1. First, GO, oxidant ammonium peroxydisulfate, and dopant acid $(\mathrm{HCl})$ in distilled water were used as the bottom aqueous layer. Aniline in toluene was used as the top organic layer. Subsequently, the organic layer was added carefully on top of the aqueous solution to form a clear organic/aqueous interface (0 h, inset in Fig. 1). Notably, it was found that a dark layer gradually formed at the interface and then thickened considerably. After standing at room temperature for 6-8 hours, the bottom aqueous layer became colorless and transparent, indicating complete consumption of GO. The GO underwent an interfacial synthesis process that can easily be

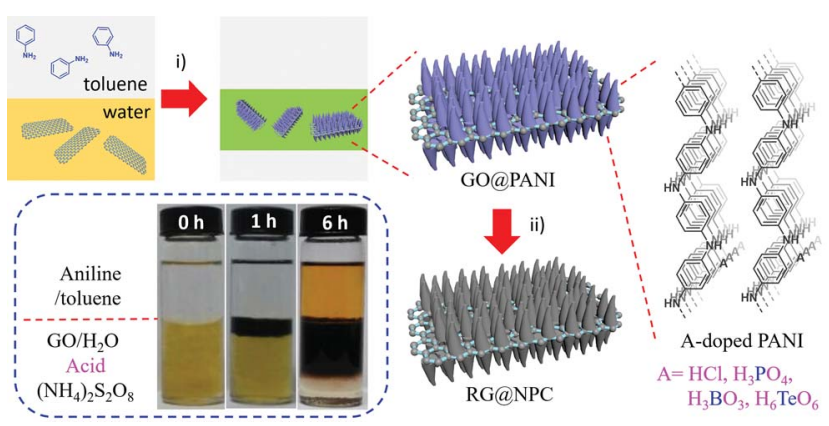

Fig. 1 Procedure for the preparation of polyaniline nanosheets and Ndoped porous carbon nanosheets. (i) Interfacial polymerization of aniline on graphene oxide surfaces at the toluene/water interface. Top: aniline in toluene; bottom: graphene oxide, acid, and ammonium persulfate in water. (ii) Pyrolysis at $1000{ }^{\circ} \mathrm{C}$ under a $5 \%\left(\mathrm{H}_{2} / \mathrm{Ar}\right)$ atmosphere. Inset: digital photos of the interfacial polymerization from 0 to $6 \mathrm{~h}$. Acid (A): hydrochloric acid, phosphoric acid, boric acid, and telluric acid. scaled up to produce GO@PANI in batches of up to $0.5 \mathrm{~kg}$ for a single preparation (Fig. S1 $\dagger$ ). At the very beginning, aniline molecules can diffuse through the interface and adsorb on GO surface, which renders the as-produced earliest nanosheets a little bit hydrophobic because the hydrophilic surface of GO would be covered with aniline due the strong electrostatic interaction between amino groups from aniline and hydrophilic groups from GO. The control experiment without GO was also performed (Fig. S2a $\dagger$ ); the digital photos of the interfacial polymerization without GO show that the dark green PANI precipitate moves downward due to the increased weight. Moreover, this process enables easy solvent recovery because of the phase separation, rendering it a highly efficient and green approach. After pyrolysis at $1000{ }^{\circ} \mathrm{C}$ under a $5 \%\left(\mathrm{H}_{2} / \mathrm{Ar}\right)$ atmosphere for $2 \mathrm{~h}$, nitrogen-doped porous carbon nanosheets (RG@NPC) can easily be produced by using GO@PANI as the precursor. The dopant $\mathrm{HCl}$ in this approach can easily be replaced with $\mathrm{H}_{3} \mathrm{PO}_{4}, \mathrm{H}_{3} \mathrm{BO}_{3}$, or $\mathrm{H}_{6} \mathrm{TeO}_{6}$ to realize different heteroatom (e.g., $\mathrm{P}, \mathrm{B}$, and $\mathrm{Te}$ ) doped porous carbon nanosheets.

To understand the interfacial process, the morphology of the as-prepared GO@PANI was imaged using scanning electron microscopy (SEM), transmission electron microscopy (TEM), and atomic force microscopy (AFM). As shown in Fig. 2a-c, numerous free-standing and highly uniform nanosheets were observed. No naked graphene nanosheets or free polyaniline nanofibers (Fig. 2h and S2b-e $\dagger$ ) were observed, which suggested that most of the aniline monomers were polymerized on the surface of the graphene. The TEM images (Fig. 2e-g) further revealed an ideal 2D sheet-like morphology as well as uniformly distributed conical PANI arrays for GO@PANI, indicating that the static reaction conditions may have caused favorable orientation of the PANI chains (striped texture ${ }^{14}$ in Fig. $2 g$ and $\mathrm{XRD}$ patterns in Fig. S3†). To reveal the loading capacity of GO, GO@PANI $(1: 10)$, GO@PANI $(1: 20)$, and GO@PANI $(1: 40)$ were fabricated using different $\mathrm{GO} /$ aniline weight ratios of $1: 10,1: 20$, and $1: 40$, respectively. As depicted in Fig. S4, $\dagger$ the 2D morphology of GO@PANI can be successfully preserved up to a ratio of $1: 40$. According to AFM analysis (Fig. $2 \mathrm{~d}$ and $55 \dagger$ ), the thicknesses of the GO@PANI (1:10), GO@PANI $(1: 20)$, and GO@PANI $(1: 40)$ are $12 \pm 3,26 \pm 5$, and $36 \pm 7 \mathrm{~nm}$,

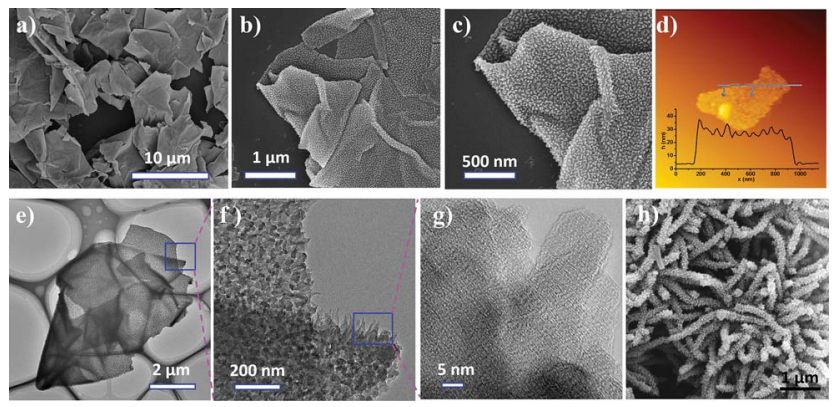

Fig. 2 Morphology of the as-prepared GO@PANI. (a-c) SEM, (d) AFM $(2.5 \mu \mathrm{m} \times 2.5 \mu \mathrm{m})$ and $(\mathrm{e}-\mathrm{g})$ TEM images of GOCPANI. (h) SEM image of PANI nanofibers prepared via the interfacial method without using $\mathrm{GO}$ in the water phase. 
respectively. Based on these analysis, such an interfacial assembly process may include three typical steps: (1) aniline molecules diffusing through the toluene/water interface and adsorbing on the GO surfaces, (2) polymerization of aniline on the GO surface in presence of oxidant and diffusing downward due to the increased weight/density, and (3) GO nanosheets and aniline molecules continuously moving upward and downward, respectively, to toluene/water interface due to the concentration gradient and electrostatic interaction between GO and aniline. In such a facile interfacial synthetic approach, not only can the uniform morphologies of the sandwich-like $2 \mathrm{D}$ nanosheets be readily obtained, but also the thicknesses can be rationally engineered by only controlling the weight ratio of graphene to aniline.

In addition to the rational control of the morphological features of GO@PANI nanosheets, the elemental composition of the nanosheets can easily be controlled by altering the composition of the aqueous layer. For example, the dopant $\mathrm{HCl}$ can be replaced by boric acid and telluric acid to produce PANI nanosheets that contain B/Te (Fig. S6 and S7†) and the corresponding heteroatom doped carbon nanosheets. Furthermore, it was found that iron cation $-\left(\mathrm{Fe}^{3+}\right)$ and phosphate anion $\left(\mathrm{PO}_{4}{ }^{3-}\right)$ anchored PANI nanosheets (GO@(Fe|PCPANI)) can easily be produced by using $\mathrm{H}_{3} \mathrm{PO}_{4}$ as an acid dopant and dissolving $\mathrm{FeCl}_{3}$ in the aqueous layer (Fig. 3a). Numerous freestanding and uniform nanosheets are evident in Fig. S8 $\dagger$ and indicate the use of different acid dopants and the introduction of transition metal ions do not affect the 2D morphology. After direct pyrolysis under a hydrogen atmosphere, RG@( $\mathrm{Fe}_{2}-$ $\mathrm{P} \subset \mathrm{NPC})$ can be easily produced by using $\mathrm{GO} @(\mathrm{Fe} \mid \mathrm{P} \subset \mathrm{PANI})$ as a precursor.

The SEM and TEM images in Fig. $3 \mathrm{~b}$ and $\mathrm{c}$ and S9† show uniform RG@( $\left.\mathrm{Fe}_{2} \mathrm{P} \subset \mathrm{NPC}\right)$ nanosheets and evenly distributed nanoparticles. Furthermore, the high-resolution TEM (HR-TEM)

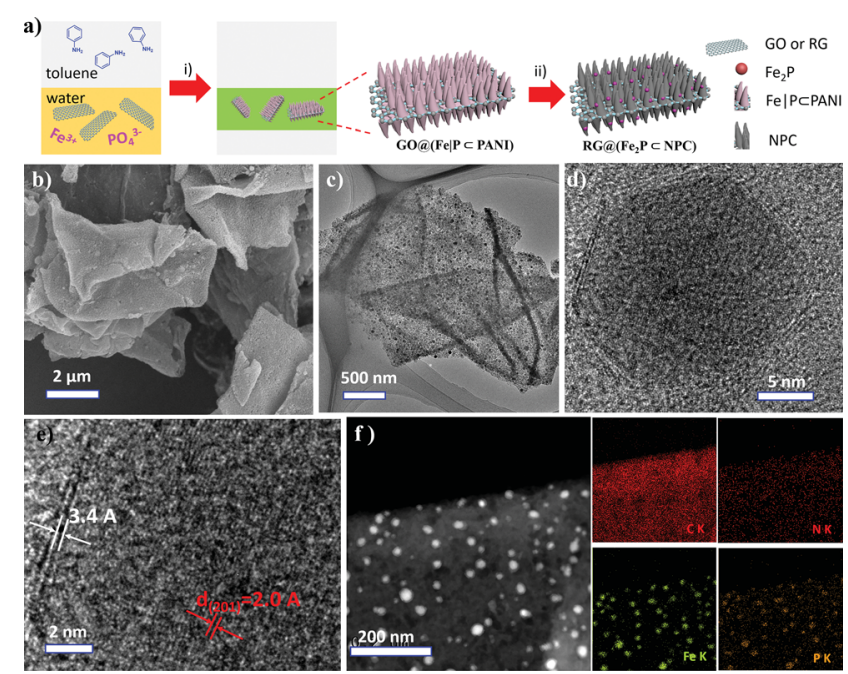

Fig. 3 (a) Schematic procedure for the preparation of metal phosphide anchored $\mathrm{N}$-doped carbon nanosheets through an interfacial approach. (b) SEM and (c) TEM images of $R G Q\left(F_{2} P \subset N P C\right)$. (d and e) $\mathrm{HR}-\mathrm{TEM}$ images of $\mathrm{RGQ}\left(\mathrm{Fe}_{2} \mathrm{P} \subset \mathrm{NPC}\right)$. (f) HAADF STEM image and elemental mapping of $\mathrm{RG}\left(\mathrm{a}\left(\mathrm{Fe}_{2} \mathrm{P} \subset \mathrm{NPC}\right)\right.$. images in Fig. 3d and e clearly show a well-resolved lattice fringe with an interplanar distance of $0.20 \mathrm{~nm}$, which can be ascribed to the (201) plane of $\mathrm{Fe}_{2} \mathrm{P}$. At the edges of the $\mathrm{Fe}_{2} \mathrm{P}$ particles, several layers of carbon with an interplanar distance of $0.34 \mathrm{~nm}$ can be observed. The high-angle annular dark-field imaging scanning TEM image (HAADF STEM) and EDX elemental mapping images of $\mathrm{C}, \mathrm{N}, \mathrm{Fe}$, and $\mathrm{P}$ for RG@( $\left.\mathrm{Fe}_{2} \mathrm{P} \subset \mathrm{NPC}\right)$ in Fig. $3 \mathrm{f}$ further verify the homogeneous distribution of the elements.

The XRD peaks of GO@(Fe $\mid \mathrm{P} \subset \mathrm{PANI})$ in Fig. 4a can be indexed to crystalline PANI, ${ }^{15}$ indicating that the introduction of ions into PANI does not affect the crystalline structure of PANI domains in the nanosheets (Fig. S3a $\dagger$ ). The XRD peaks of RG@(Fe $\left.{ }_{2} \mathrm{P} \subset \mathrm{NPC}\right)$ in Fig. 4 a can be indexed to crystalline $\mathrm{Fe}_{2} \mathrm{P}$ with lattice constants $\alpha=5.867 \AA$ and $c=3.458 \AA$ (JCPDS no. 51-0943). The intense peaks located at 40.2, 44.1, 47.2, 52.8, $54.0,54.6,73.6$, and $79.0^{\circ}$ can be assigned to the (111), (201), (210), (002), (300), (211), (212), and (302) crystal planes, demonstrating that phase-pure $\mathrm{Fe}_{2} \mathrm{P}$ was successfully obtained after pyrolysis. Furthermore, a pronounced characteristic diffraction peak located at $25.2^{\circ}$ was observed in the XRD spectrum of RG@( $\left.\mathrm{Fe}_{2} \mathrm{P} \subset \mathrm{NPC}\right)$, which can be ascribed to amorphous porous carbon. For comparison, pure $\mathrm{Fe}_{2} \mathrm{P}$ nanoparticles were also synthesized (Fig. S10 and S11†).

The X-ray photoelectron spectroscopy (XPS) spectra of $\mathrm{RG} @\left(\mathrm{Fe}_{2} \mathrm{P} \subset \mathrm{NPC}\right)$ were measured to confirm the chemical composition and valence states. The XPS survey scans (Fig. S12a †) reveal the presence of Fe, P, N, O, and C elements. The Fe $2 p_{3 / 2}$ and Fe $2 p_{1 / 2}$ couple peaks located at 707.1/720.2 eV and $711.2 / 724.0 \mathrm{eV}$ can be ascribed to $\mathrm{Fe}_{2} \mathrm{P}$ and surface-oxidized ion species, respectively (Fig. S12b $\dagger$ ). The $\mathrm{P} 2 \mathrm{p} 1 / 2$ and $\mathrm{P} 2 \mathrm{p} 3 / 2$ peaks at 130.2 and $129.3 \mathrm{eV}$ can be attributed to $\mathrm{Fe}_{2} \mathrm{P}$, and the $\mathrm{P}$ $2 \mathrm{p}$ peak at $133.3 \mathrm{eV}$ can be attributed to residual phosphate (Fig. S12c $\dagger$ ). This result is consistent with the result for the pure $\mathrm{Fe}_{2} \mathrm{P}$ nanoparticles that we prepared by a similar method (Fig. S12 $\dagger$ ). The N (2.59\%), Fe (3.71\%), and P (3.35\%) contents based on XPS analysis for $\mathrm{RG} @\left(\mathrm{Fe}_{2} \mathrm{P} \subset \mathrm{NPC}\right)$ are summarized in Table S1. $\dagger$ The nitrogen physisorption measurement indicates

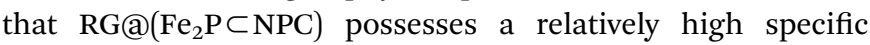
surface area of $240 \mathrm{~m}^{2} \mathrm{~g}^{-1}$ and a hierarchically porous structure (both micropores less than $2 \mathrm{~nm}$ and mesopores between 2 and $50 \mathrm{~nm}$ ) in comparison with NPC and RG@NPC (Fig. 4b and S13; Table S2 $\dagger$ ). Furthermore, RG@(MoP $\subset \mathrm{NPC}), \mathrm{RG} @\left(\mathrm{Co}_{2} \mathrm{P} \subset \mathrm{NPC}\right)$, and RG@( $\mathrm{Ni}_{12} \mathrm{P}_{5} \subset \mathrm{NPC}$ ) (Fig. $\mathrm{S} 14-\mathrm{S} 16 \dagger$ ) were successfully prepared by means of only replacing $\mathrm{FeCl}_{3}$ by $\left(\mathrm{NH}_{4}\right)_{2} \mathrm{Mo}_{4} \mathrm{O}_{13}$,
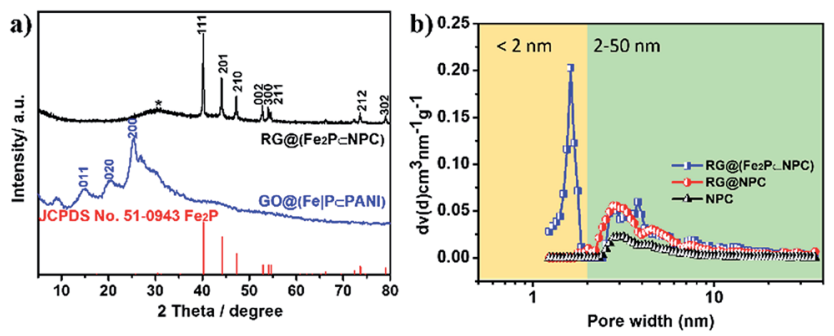

Fig. 4 (a) XRD patterns of $R G\left(\left(F_{2} P \subset N P C\right), G O @(F e \mid P \subset P A N I)\right.$ and the diffraction peaks referring to JCPDS card number 51-0943. (b) Pore size distribution of the samples using the DFT method. 
$\mathrm{CoCl}_{2}$, and $\mathrm{Ni}(\mathrm{OAc})_{2}$, respectively, indicating the versatility of this procedure regarding different MP-embedded carbon nanosheets. All these results demonstrate that versatile MPs particle-anchored NPC can easily be fabricated and that the 2D morphology, crystalline character of the MPs, inorganic heterostructures, ${ }^{16}$ and hierarchical porous features of the nanosheets can be controlled rationally.

Integration of the multiple advantages of nitrogen doping, hierarchically porous structure, nanosheet morphology and carbon-wall-wrapped metal phosphides in such kinds of the asprepared 2D hybrid materials holds promise for their application in electrochemical capacitors. $\mathrm{RG} @\left(\mathrm{Fe}_{2} \mathrm{P} \subset \mathrm{NPC}\right)$ was chosen as a typical example for electrochemical capacitor study. First, the cyclic voltammograms (CVs) of $\mathrm{RG} @\left(\mathrm{Fe}_{2} \mathrm{P} \subset \mathrm{NPC}\right)$ were measured at a sweep rate of $5 \mathrm{mV} \mathrm{s}^{-1}$ in $6 \mathrm{M} \mathrm{KOH}$ and were compared to the CVs of NPC, RG@NPC, and $\mathrm{Fe}_{2} \mathrm{P}$ (Fig. 5a, S17a and S18a-c $\dagger$ ). The CVs of RG@NPC and NPC showed similar current densities, whereas the curve of $\mathrm{RG} @\left(\mathrm{Fe}_{2} \mathrm{P} \subset \mathrm{NPC}\right)$ displayed a much larger current density and typical redox current peaks corresponding to reversible reactions between $\mathrm{Fe}^{2+}$ and $\mathrm{Fe}^{3+}$. Based on the relevant literature, ${ }^{17}$ the possible reaction corresponding to the redox peaks is as following:

$$
\begin{gathered}
\mathrm{Fe}^{2+}+2 \mathrm{OH}^{-} \rightarrow \mathrm{Fe}(\mathrm{OH})_{2} \\
\mathrm{Fe}(\mathrm{OH})_{2}+\mathrm{OH}^{-} \leftrightarrow \mathrm{FeOOH}+\mathrm{H}_{2} \mathrm{O}+\mathrm{e}^{-}
\end{gathered}
$$

The galvanostatic charge/discharge curves were measured at a current density of $1-10 \mathrm{~A} \mathrm{~g}^{-1}$ (Fig. 5b, S17b and S18d-f $f^{\dagger}$ ). The specific capacitance of $\mathrm{RG} @\left(\mathrm{Fe}_{2} \mathrm{P} \subset \mathrm{NPC}\right)$ was calculated to be as high as $1098 \mathrm{~F} \mathrm{~g}^{-1}$, which is more than 10 times the capacitance values of NPC $\left(90 \mathrm{~F} \mathrm{~g}^{-1}\right)$, RG@NPC $\left(106 \mathrm{~F} \mathrm{~g}^{-1}\right)$, and $\mathrm{Fe}_{2} \mathrm{P}(44.3$ $\mathrm{F} \mathrm{g}^{-1}$ ) at $1 \mathrm{~A} \mathrm{~g}^{-1}$ (Fig. 5c). RG@( $\mathrm{Fe}_{2} \mathrm{P} \subset \mathrm{NPC}$ ) exhibited a high performance rate capability (Fig. 5c) and an extremely high
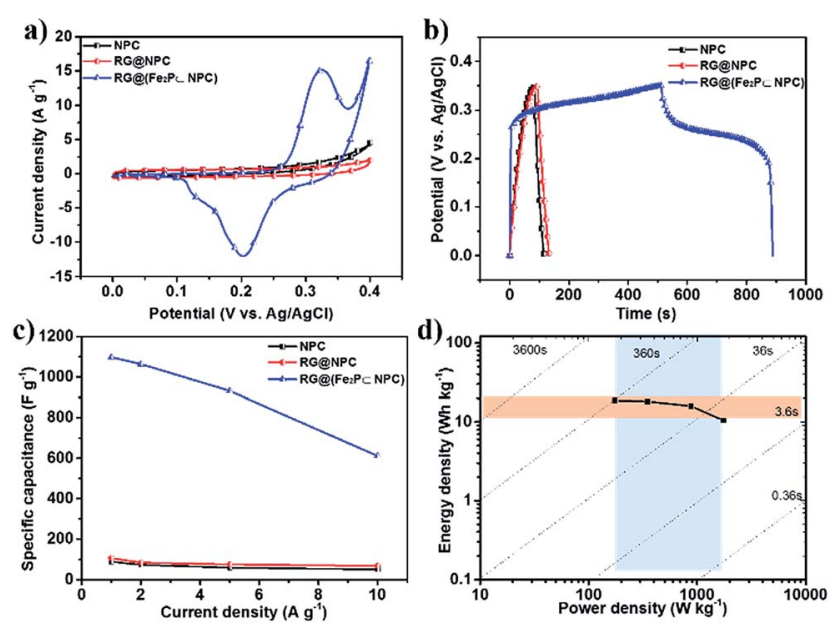

Fig. 5 (a) Cyclic voltammograms and (b) galvanostatic chargedischarge curves of NPC, RG@NPC and RG@(Fe 2 P $\subset N P C)$ obtained in $6 \mathrm{M} \mathrm{KOH}$ at $5 \mathrm{mV} \mathrm{s}^{-1}$ and the current density of $1.0 \mathrm{~A} \mathrm{~g}^{-1}$ respectively. (c) Specific capacitance of NPC, RGQNPC and RGQ(Fe $\left.{ }_{2} P \subset N P C\right)$ at different current densities $\left(1.0-10 \mathrm{~A} \mathrm{~g}^{-1}\right)$. (d) Ragone plot of the supercapacitor based on $\mathrm{RGQ}\left(\mathrm{Fe}_{2} \mathrm{P} \subset \mathrm{NPC}\right)$. specific capacitance of $611 \mathrm{~F} \mathrm{~g}^{-1}$ at $10 \mathrm{~A} \mathrm{~g}^{-1}$, outperforming state-of-the-art performance among porous carbon and MPbased supercapacitors (Table S3 $\uparrow) .{ }^{18}$ Furthermore, RG@( $\mathrm{Fe}_{2}-$ $\mathrm{P} \subset \mathrm{NPC}$ ) showed excellent cycling stability with $77.9 \%$ capacitance retention after 1000 cycles (Fig. S17c †). Energy and power densities are critical parameters for energy storage devices. Fig. $5 d$ presents the Ragone plot of $\mathrm{RG} @\left(\mathrm{Fe}_{2} \mathrm{P} \subset \mathrm{NPC}\right)$ related to energy and power densities. The supercapacitor based on $\mathrm{RG} @\left(\mathrm{Fe}_{2} \mathrm{P} \subset \mathrm{NPC}\right)$ exhibited a maximum energy density of 18.7 $\mathrm{W} \mathrm{kg}^{-1}$ at $175.1 \mathrm{~W} \mathrm{~kg}^{-1}$, which is comparable to the maximum energy densities of most heteroatom-doped carbon-based electrodes. ${ }^{19}$ Nyquist plots and equivalent circuits (Fig. S19 and Table S4 $\dagger$ ) of these electrodes reveal that RG@( $\left.\mathrm{Fe}_{2} \mathrm{P} \subset \mathrm{NPC}\right)$ exhibited the lowest charge-transfer resistance $(0.25 \Omega)$ among the as-prepared materials (RG@NPC: $0.45 \Omega$, NPC: $0.61 \Omega, \mathrm{Fe}_{2} \mathrm{P}$ : $1032 \Omega$ ), benefiting from the high conductivity of porous carbons and the long-distance conductivity of graphene layers. Thus, the good conductivity, hierarchically porous structure, and the synergistic effect between $\mathrm{Fe}_{2} \mathrm{P}$ and NPC may be effectively combined together to contribute to the outstanding supercapacitor performance..$^{20}$

MPs have recently been widely investigated because of their potential for the high performance electrochemically catalyzed hydrogen evolution reaction (HER). ${ }^{21}$ Therefore, the electrocatalytic HER activity of the obtained RG@(MP $\subset$ NPC)s in $0.5 \mathrm{M}$ $\mathrm{H}_{2} \mathrm{SO}_{4}$ was examined. The iR correction was applied to all electrochemical measurements because of ohmic resistance during measurement. The polarization curves and calculated Tafel slopes of the as-prepared materials are depicted in Fig. S20a and b. $\dagger$ Table S6 $\dagger$ shows that RG@(Fe $\left.{ }_{2} \mathrm{P} \subset \mathrm{NPC}\right)$ exhibited the lowest onset overpotential $(45 \mathrm{mV})$, the lowest overpotentials $\left(102 \mathrm{mV}\right.$ at $10 \mathrm{~mA} \mathrm{~cm}{ }^{-2} ; 191 \mathrm{mV}$ at $100 \mathrm{~mA}$ $\mathrm{cm}^{-2}$ ), and the smallest Tafel slope $\left(61 \mathrm{mV} \mathrm{dec}^{-1}\right)$ thereby suggesting that the HER occurs on the surface of RG@( $\mathrm{Fe}_{2^{-}}$ $\mathrm{P} \subset \mathrm{NPC}$ ) according to the Volmer-Heyrovsky mechanism..$^{22}$ The polarization curves (Fig. S20d $\dagger$ ) of the $\mathrm{RG} @\left(\mathrm{Fe}_{2} \mathrm{P} \subset \mathrm{NPC}\right)$ cathode before and after accelerated degradation testing for 3000 continuous cycles (sweeping the potential in a range of $-500 \mathrm{mV}$ to $+200 \mathrm{mV}$ versus RHE at a rate of $100 \mathrm{mV} \mathrm{s}^{-1}$ ). The electrode exhibited minimal degradation after 3000 cycles, with an overpotential increase of only $10 \pm 1 \mathrm{mV}$, to achieve current densities of 10 and $100 \mathrm{~mA} \mathrm{~cm}{ }^{-2}$, respectively. These results were comparable to those reported for $\mathrm{Fe}_{2} \mathrm{P}$-based catalysts and other MP-based catalysts (Table S7 $\dagger$ ). ${ }^{23}$ The superior HER performance of $\mathrm{RG} @\left(\mathrm{Fe}_{2} \mathrm{P} \subset \mathrm{NPC}\right)$ compared to that of RG@(MP $\subset$ NPC) can be further assessed by electrochemical impedance spectroscopy (Fig. S20c $\dagger$ ) and calculated chargetransfer resistance (Table S5 $\dagger$ ) based on a two-time-constant model (Fig. S21 $\dagger$ ). The HER performance of the as-prepared materials can be further optimized by controlling different parameters such as pyrolysis temperature, particle size, and MP content.

Bimetallic nanoparticle-based nanomaterials have exhibited excellent potential as efficient electrochemical catalysts for the HER, ${ }^{24} \mathrm{CO}_{2}$ reduction reaction, ${ }^{25}$ oxygen evolution reaction $(\mathrm{OER}){ }^{26}$ and oxygen reduction reaction (ORR). ${ }^{27}$ As a proof-ofconcept, $\mathrm{Fe}_{2} \mathrm{P}$ and $\mathrm{Co}_{2} \mathrm{P}$ co-embedded 2D NPC (denoted as 
$\left.\mathrm{RG} @\left(\mathrm{Fe}_{2} \mathrm{P} \mid \mathrm{Co}_{2} \mathrm{P} \subset \mathrm{NPC}\right)\right)$ was further prepared through the same procedure by adding both $\mathrm{FeCl}_{3}$ and $\mathrm{CoCl}_{2}$ to the aqueous layer. The HR-TEM image in Fig. 6a clearly shows a wellresolved lattice fringe with interplanar distances of $0.20 \mathrm{~nm}$ and $0.44 \mathrm{~nm}$, corresponding to the (201) plane of $\mathrm{Fe}_{2} \mathrm{P}$ and the (101) plane of $\mathrm{Co}_{2} \mathrm{P}$, respectively. This is consistent with the XRD pattern and XPS result of $\mathrm{RG} @\left(\mathrm{Fe}_{2} \mathrm{P} \mid \mathrm{Co}_{2} \mathrm{P} \subset \mathrm{NPC}\right)$ (Fig. S22 and S23†). The HAADF STEM image and EDX elemental mapping further illustrate that $\mathrm{C}, \mathrm{N}, \mathrm{Fe}, \mathrm{Co}$, and $\mathrm{P}$ are uniformly distributed in the bimetallic phosphide-based 2D hybrid (Fig. 6b).

The electrocatalytic properties of $\mathrm{RG}(\mathrm{MP} \subset \mathrm{NPC})$ for the ORR was subsequently evaluated under alkaline conditions (0.1 $\mathrm{M} \mathrm{KOH})$. First, the CV of RG@ $\left(\mathrm{Fe}_{2} \mathrm{P} \mid \mathrm{Co}_{2} \mathrm{P} \subset \mathrm{NPC}\right)$ in $\mathrm{O}_{2}$-saturated $0.1 \mathrm{M} \mathrm{KOH}$ revealed an ORR peak potential of $0.70 \mathrm{~V}$ versus RHE (Fig. 7a), which is 5-6 mV higher than those of RG@NPC and single MP-embedded 2D NPC (Fig. S24 $\dagger$ ). According to the LSV curves under $1600 \mathrm{rpm}$ (Fig. 7b), RG@( $\left(\mathrm{Fe}_{2} \mathrm{P} \mid \mathrm{Co}_{2} \mathrm{P} \subset \mathrm{NPC}\right)$ showed an excellent onset potential of $0.8 \mathrm{~V}$ and a half-wave potential of $0.78 \mathrm{~V}$, which are superior to those of $\mathrm{RG} @\left(\mathrm{Fe}_{2^{-}}\right.$ $\mathrm{P} \subset \mathrm{NPC})$ and $\mathrm{RG}\left(\mathrm{Co}_{2} \mathrm{P} \subset \mathrm{NPC}\right)$. The half-wave potential difference between RG@ $\left(\mathrm{Fe}_{2} \mathrm{P} \mid \mathrm{Co}_{2} \mathrm{P} \subset \mathrm{NPC}\right)$ and $\mathrm{Pt} / \mathrm{C}$ was found to be only $49 \mathrm{mV}$ (Fig. S26b $\dagger$ ). According to the rotating ringdisk electrode experiment (Fig. S26a†), a four-electron transfer
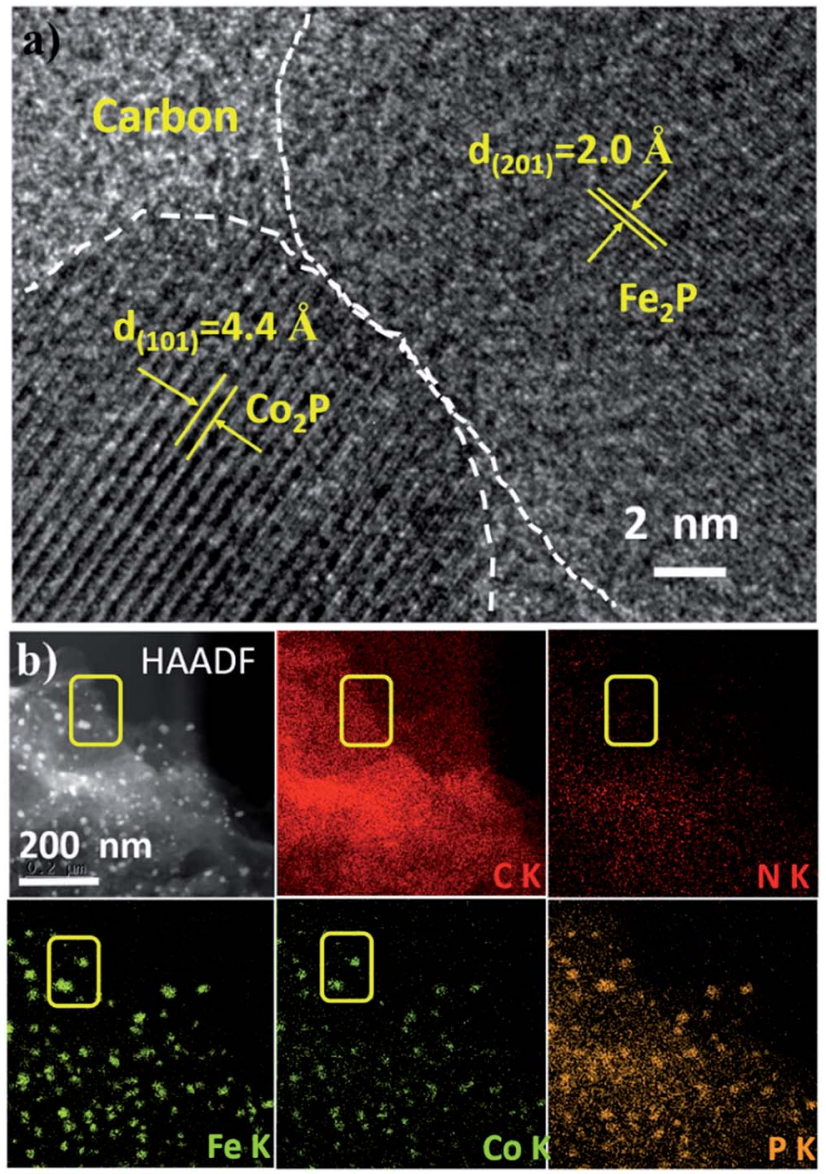

Fig. 6 (a) High resolution TEM image, (b) HAADF STEM image and elemental mapping of $\mathrm{RGQ}\left(\mathrm{CO}_{2} \mathrm{P} \mid \mathrm{Fe}_{2} \mathrm{P} \subset \mathrm{NPC}\right)$.
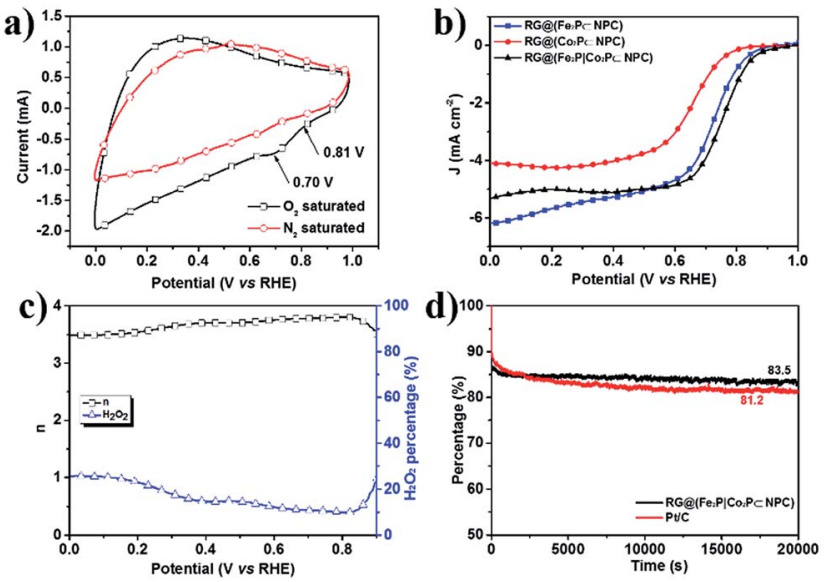

Fig. 7 (a) Cyclic voltammograms of $\mathrm{RG}\left(\mathrm{CO}_{2} \mathrm{P} \mid \mathrm{Fe}_{2} \mathrm{P} \subset \mathrm{NPC}\right)$ in $\mathrm{N}_{2}-$ and $\mathrm{O}_{2}$-saturated $0.1 \mathrm{M} \mathrm{KOH}$. (b) LSV curves of $\mathrm{RGQ}\left(\mathrm{CO}_{2} \mathrm{P} \mid \mathrm{Fe}_{2} \mathrm{P} \subset \mathrm{NPC}\right)$, $\mathrm{RG}\left(\mathrm{CO}_{2} \mathrm{P} \subset \mathrm{NPC}\right), \mathrm{RGQ}\left(\mathrm{Fe}_{2} \mathrm{P} \subset \mathrm{NPC}\right)$ in $\mathrm{O}_{2}$-saturated $0.1 \mathrm{M} \mathrm{KOH}$ solution at $1600 \mathrm{rpm}$ at a scan rate of $5 \mathrm{mV} \mathrm{s}^{-1}$. (c) Calculated electrontransfer number $(n)$ and percentage of $\mathrm{H}_{2} \mathrm{O}_{2}(\%)$ for $\mathrm{RGQ}\left(\mathrm{CO}_{2} \mathrm{P} \mid \mathrm{Fe}_{2}-\right.$ $P \subset N P C)$ as a function of the electrode potential. (d) Current-time $(i-t)$ chronoamperometric response of $\mathrm{RG}\left(\mathrm{Fe}_{2} \mathrm{P} \mid \mathrm{CO}_{2} \mathrm{P} \subset \mathrm{NPC}\right)$ and $\mathrm{Pt} / \mathrm{C}$ at $-0.4 \mathrm{~V}$.

$\left(n \approx 3.78\right.$ at $0.7 \mathrm{~V}$ ) mechanism with a low yield of $\mathrm{H}_{2} \mathrm{O}_{2}$ (Fig. 7c) can be calculated (eqn (1) and (2)). In addition, the durability of $\mathrm{RG} @\left(\mathrm{Fe}_{2} \mathrm{P} \mid \mathrm{Co}_{2} \mathrm{P} \subset \mathrm{NPC}\right)$ was tested at a constant voltage of 0.45 $\mathrm{V}$ for $20000 \mathrm{~s}$ (Fig. 7d). Notably, it exhibited a high relative current $(83.5 \%)$ that persisted after $20000 \mathrm{~s}$, which is higher than that of $\mathrm{Pt} / \mathrm{C}(81.2 \%)$. This may attributed to the fact that the MPs were well wrapped by the carbon layer. Compared with the ORR performance of the as-prepared single MP-anchored

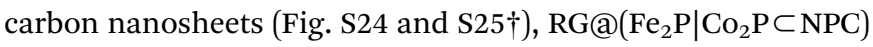
showed the best ORR activity. This may attributed to the synergistic effect between bimetallic nanoparticles. Generally, the superior electrocatalytic activity of the as-prepared RG@(MPs $\subset$ NPC)s are attributable to the following causes: (1) the small and uniformly distributed crystallized MPs produce large active sites for the HER and ORR; (2) the 2D morphology and strong interactions between the RG, NPC, and MPs substantially increase the electrical conductivity, thereby enhancing electron transfer.

\section{Conclusions}

In summary, we demonstrated an interfacial engineering method for the rational synthesis of 2D PANI nano-array and corresponding nitrogen-doped porous carbon nanosheets. Not only was it easy to produce a sandwich-like 2D morphology, but also the thickness, anchored cations and anions, and different metal phosphides were easily and rationally engineered by controlling the composition of the aqueous layer. The novel structural features of these hybrids enabled outstanding electrochemical performance. We believe that this interfacial approach can be extended to the controllable synthesis of various $2 \mathrm{D}$ materials coupled sandwich-like hybrid materials with potential application in a wide range of areas. Such an 
interfacial method can be used to fabricate various types of heteroatom-doped porous carbon nanosheets and MPanchored carbon nanosheets. Moreover, it can be used to produce versatile nanoparticles, such as transition metal carbide (Fig. S27†) anchored porous carbon nanosheets for a broad range of applications in the field of energy storage and conversion.

\section{Acknowledgements}

C. L. and S. L. contributed equally to this work. This work was financially supported by the 973 Programs of China (2013CBA01602), National Natural Science Foundation of China (51403126 and 61306018), the Shanghai Committee of Science and Technology (15JC1490500), Shanghai Municipal Natural Science Foundation (16JC1400703), ERC Grant on 2DMATER, UP-GREEN and EU Graphene Flagship.

\section{Notes and references}

1 (a) A. G. MacDiarmid, Angew. Chem., Int. Ed., 2001, 40, 2581; (b) O. Bubnova, Z. U. Khan, A. Malti, S. Braun, M. Fahlman, M. Berggren and X. Crispin, Nat. Mater., 2011, 10, 429; (c) J. E. Yoo, K. S. Lee, A. Garcia, J. Tarver, E. D. Gomez, K. Baldwin, Y. Sun, H. Meng, T. Q. Nguyen and Y. L. Loo, Proc. Natl. Acad. Sci. U. S. A., 2010, 107, 5712; (d) J. Heinze, B. A. Frontana-Uribe and S. Ludwigs, Chem. Rev., 2010, 110, 4724.

2 (a) J. Huang and R. B. Kaner, J. Am. Chem. Soc., 2004, 126, 851; (b) J. C. Michaelson and A. J. McEvoy, J. Chem. Soc., Chem. Commun., 1994, 79.

3 (a) G. D. Fu, J. P. Zhao, Y. M. Sun, E. T. Kang and K. G. Neoh, Macromolecules, 2007, 40, 2271; (b) P. Anilkumar and M. Jayakannan, Macromolecules, 2008, 41, 7706.

4 J. Huang, S. Virji, B. H. Weiller and R. B. Kaner, J. Am. Chem. Soc., 2003, 125, 314.

5 L. Zhang, Z. D. Zujovic, H. Peng, G. A. Bowmaker, P. A. Kilmartin and J. Travas-Sejdic, Macromolecules, 2008, 41, 8877.

6 S. Li, D. Wu, C. Cheng, J. Wang, F. Zhang, Y. Su and X. Feng, Angew. Chem., Int. Ed., 2013, 52, 12105.

7 (a) Z. Wei, Z. Zhang and M. Wan, Langmuir, 2002, 18, 917; (b) Z. Wei and M. Wan, J. Appl. Polym. Sci., 2003, 87, 1297; (c) H. Qiu, M. Wan, B. Matthews and L. Dai, Macromolecules, 2001, 34, 675.

8 (a) Q. Hao, H. Wang, X. Yang, L. Lu and X. Wang, Nano Res., 2011, 4, 323-333; (b) J. Zhu, M. Chen, H. Qu, X. Zhang, H. Wei, Z. Luo, H. A. Colorado, S. Wei and Z. Guo, Polymer, 2012, 53, 5953-5964; (c) Y. Jin, S. Huang, M. Zhang and M. Jia, Synth. Met., 2013, 168, 58-64.

9 (a) X. Zhuang, Y. Mai, D. Wu, F. Zhang and X. Feng, Adv. Mater., 2015, 27, 403; (b) X. Zhuang, F. Zhang, D. Wu, N. Forler, H. Liang, M. Wagner, D. Gehrig, M. R. Hansen, F. Laquai and X. Feng, Angew. Chem., Int. Ed., 2013, 52, 9668; (c) X. Zhuang, F. Zhang, D. Wu and X. Feng, Adv. Mater., 2014, 26, 3081; (d) X. Zhuang, D. Gehrig, N. Forler, H. Liang, M. Wagner, M. R. Hansen, F. Laquai, F. Zhang and X. Feng, Adv. Mater., 2015, 27, 3789; (e) S. Liu, J. Zhang, R. Dong, P. Gordiichuk, T. Zhang, X. Zhuang, Y. Mai, F. Liu, A. Herrmann and X. Feng, Angew. Chem., Int. Ed., 2016, 55, 12516; (f) K. Yuan, X. Zhuang, H. Fu, G. Brunklaus, M. Forster, Y. Chen, X. Feng and U. Scherf, Angew. Chem., Int. Ed., 2016, 55, 6858; (g) Y. Su, Z. Yao, F. Zhang, H. Wang, Z. Mics, E. Cánovas, M. Bonn, X. Zhuang and X. Feng, Adv. Funct. Mater., 2016, 26, 5893-5902.

10 (a) S. Najmaei, Z. Liu, W. Zhou, X. Zou, G. Shi, S. Lei, B. I. Yakobson, J.-C. Idrobo, P. M. Ajayan and J. Lou, Nat. Mater., 2013, 12, 754; (b) S. Ithurria, M. Tessier, B. Mahler, R. Lobo, B. Dubertret and A. L. Efros, Nat. Mater., 2011, 10, 936; (c) X. Huang, X. Qi, F. Boey and H. Zhang, Chem. Soc. Rev., 2012, 41, 666.

11 (a) H. Wang, J. T. Robinson, G. Diankov and H. Dai, J. Am. Chem. Soc., 2010, 132, 3270; (b) S. Chen, J. Zhu, X. Wu, Q. Han and X. Wang, ACS Nano, 2010, 4, 2822; (c) J. Y. Son, Y.-H. Shin, H. Kim and H. M. Jang, ACS Nano, 2010, 4, 2655; (d) C. X. Guo, H. B. Yang, Z. M. Sheng, Z. S. Lu, Q. L. Song and C. M. Li, Angew. Chem., Int. Ed., 2010, 49, 3014; (e) A. Cao, Z. Liu, S. Chu, M. Wu, Z. Ye, Z. Cai, Y. Chang, S. Wang, Q. Gong and Y. Liu, Adv. Mater., 2010, 22, 103; $(f)$ Z. Huang, C. Lv, Z. Chen, Z. Chen, F. Tian and C. Zhang, Nano Energy, 2015, 12, 666.

12 (a) P. S. Toth, M. Velický, M. A. Bissett, T. J. A. Slater, N. Savjani, A. K. Rabiu, A. M. Rakowski, J. R. Brent, S. J. Haigh, P. O'Brien and R. A. W. Dryfe, Adv. Mater., 2016, 28, 8256-8264; (b) P. S. Toth, Q. M. Ramasse, M. Velicky and R. A. W. Dryfe, Chem. Sci., 2015, 6, 1316-1323. 13 (a) F. Bonaccorso, L. Colombo, G. Yu, M. Stoller, V. Tozzini, A. C. Ferrari, R. S. Ruoff and V. Pellegrini, Science, 2015, 347, 1246501; (b) S. Eigler and A. Hirsch, Angew. Chem., Int. Ed., 2014, 53, 7720.

14 L. Mazerolles, S. Folch and P. Colomban, Macromolecules, 1999, 32, 8504.

15 J. P. Pouget, M. E. Jozefowicz, A. J. Epstein, X. Tang and A. G. MacDiarmid, Macromolecules, 1991, 24, 779.

16 P. S. Toth, M. Velický, M. A. Bissett, T. J. Slater, N. Savjani, A. K. Rabiu, A. M. Rakowski, J. R. Brent, S. J. Haigh and P. O'Brien, Adv. Mater., 2016, 28, 8256-8264.

17 (a) D. Wang, L. B. Kong, M. C. Liu, Y. C. Luo and L. Kang, Chem.-Eur. J., 2015, 21, 17897-17903; (b) X. Chen, M. Cheng, D. Chen and R. Wang, ACS Appl. Mater. Interfaces, 2016, 8, 3892-3900.

18 (a) J. Chang, M. Jin, F. Yao, T. H. Kim, V. T. Le, H. Yue, F. Gunes, B. Li, A. Ghosh, S. Xie and Y. H. Lee, Adv. Funct. Mater., 2013, 23, 5074; (b) Z. Lei, L. Lu and X. S. Zhao, Energy Environ. Sci., 2012, 5, 6391; (c) A. Sumboja, C. Y. Foo, X. Wang and P. S. Lee, Adv. Mater., 2013, 25, 2809; (d) J. Xu, K. Wang, S.-Z. Zu, B.-H. Han and Z. Wei, ACS Nano, 2010, 4, 5019; (e) K. Yuan, Y. Xu, J. Uihlein, G. Brunklaus, L. Shi, R. Heiderhoff, M. Que, M. Forster, T. Chasse, T. Pichler, T. Riedl, Y. Chen and U. Scherf, Adv. Mater., 2015, 27, 6714.

19 (a) Q. Wang, J. Yan and Z. J. Fan, Energ Environ. Sci., 2016, 9, 729; (b) L. Liu, Z. Niu and J. Chen, Chem. Soc. Rev., 2016, 45, 4340 . 
20 (a) S. He, H. Hou and W. Chen, J. Power Sources, 2015, 280, 678-686; (b) S. He and W. Chen, Nanoscale, 2015, 7, 69576990; (c) J. Masa, W. Xia, M. Muhler and W. Schuhmann, Angew. Chem., Int. Ed., 2015, 54, 10102.

21 (a) Y. Shi and B. Zhang, Chem. Soc. Rev., 2016, 45, 1529; (b) M. Sun, H. Liu, J. Qu and J. Li, Adv. Energy Mater., 2016, 6, 1600087.

22 B. E. Conway and B. V. Tilak, Electrochim. Acta, 2002, 47, 3571.

23 (a) Q. Liu, J. Q. Tian, W. Cui, P. Jiang, N. Y. Cheng, A. M. Asiri and X. P. Sun, Angew. Chem., Int. Ed., 2014, 53, 6710; (b) P. Xiao, M. A. Sk, L. Thia, X. M. Ge, R. J. Lim, J. Y. Wang, K. H. Lim and X. Wang, Energy Environ. Sci., 2014, 7, 2624; (c) Z. C. Xing, Q. Liu, A. M. Asiri and X. P. Sun, Adv. Mater., 2014, 26, 5702 .

24 (a) Y. Tan, H. Wang, P. Liu, Y. Shen, C. Cheng, A. Hirata, T. Fujita, Z. Tang and M. Chen, Energy Environ. Sci., 2016,
9, 2257; (b) Z. F. Huang, J. Song, K. Li, M. Tahir, Y. T. Wang, L. Pan, L. Wang, X. Zhang and J. J. Zou, J. Am. Chem. Soc., 2016, 138, 1359; (c) Q. Lu, G. S. Hutchings, W. Yu, Y. Zhou, R. V. Forest, R. Tao, J. Rosen, B. T. Yonemoto, Z. Cao, H. Zheng, J. Q. Xiao, F. Jiao and J. G. Chen, Nat. Commun., 2015, 6, 6567.

25 (a) S. Rasul, D. H. Anjum, A. Jedidi, Y. Minenkov, L. Cavallo and K. Takanabe, Angew. Chem., Int. Ed., 2015, 54, 2146; (b) X. Sun, Q. Zhu, X. Kang, H. Liu, Q. Qian, Z. Zhang and B. Han, Angew. Chem., Int. Ed., 2016, 55, 6771.

26 (a) R. Zhang, C. Zhang and W. Chen, J. Mater. Chem. A, 2016, 4, 18723; (b) B. Ni and X. Wang, Chem. Sci., 2015, 6, 3572.

27 (a) Y.-Z. Chen, C. Wang, Z.-Y. Wu, Y. Xiong, Q. Xu, S.-H. Yu and H.-L. Jiang, Adv. Mater., 2015, 27, 5010; (b) Z. Li, M. Shao, L. Zhou, Q. Yang, C. Zhang, M. Wei, D. G. Evans and X. Duan, Nano Energy, 2016, 25, 100. 